\title{
The Effects of Surfaces on Dynamic Critical Behavior
}

\author{
S. Dietrich and H.W. Diehl
}

Sektion Physik der Universität, München, Federal Republic of Germany

Received March 23, 1983

Z. Phys. B - Condensed Matter 51, 343-354 (1983)

1. The last sentence of Sect.IV should read: One finds again an exponential large-distance decay

$W_{R}^{(0,1 ; 1,0)} \sim \exp \left(-x^{2} / \xi_{t}^{2}\right) \quad$ with $\xi_{t} \sim(\lambda t)^{1 / z}$

and a long-time tail $\sim t^{-1-2 \beta_{1 / v z}}$.

2. In the second line below (V.1): replace $\Theta$ by $\Theta_{11}$.

3. The sentence below (V.4) should read: To order

$\varepsilon^{0}, \Psi_{11}$ behaves as $1-\bar{\omega}^{2} / 8+O\left(\bar{\omega}^{4}\right)$ at small $\bar{\omega}$ and as

const $\cdot \bar{\omega}^{-1-\gamma_{11}^{\text {ord } / v z}}=\mathrm{const} \cdot \bar{\omega}^{-1 / 2+O(\varepsilon)}$

at large $\bar{\omega}$.
4. The last line of Appendix A should read $\omega_{1}=$ $-\sum_{j \neq 1} \omega_{j}$.

5. In (B.19): replace $\Theta$ by $\Theta_{11}$.

6. Equation (B.20) should read:

$y_{ \pm}=2^{-1}\left\{\left[\left(1+\not^{-2}\right)^{2}+\bar{\omega}^{2}\right]^{1 / 2} \pm\left(1+\not^{-2}\right)\right\}$.

S. Dietrich

H.W. Diehl

Sektion Physik

Universität München

Theresienstrasse 37

D-8000 München 2

Federal Republic of Germany 